\title{
Synthesis of scalable and tunable slightly oxidized graphene via Chemical Vapor Deposition
}

Rizwan Ur Rehman Sagar ${ }^{1,2}$, Florian J. Stadler ${ }^{1, *}$ Mina Namvari ${ }^{1,2}$, and Sachin T. Navale ${ }^{1,2}$,

\begin{abstract}
Semiconducting, large sheets of carbon as an active material in optoelectronic research are missing and reduced graphene oxide (rGO) can be a good candidate. However, chemical synthesis cannot produce large sheets of rGO (i.e. maximum: $20 \sim 30 \mu \mathrm{m}$ ) as well as high quality rGO due to the restraints of fabrication method. Thus, a novel strategy for the synthesis of large sheets of semiconducting rGO is urgently required. Large area slightly oxidized graphene ( $\mathrm{SOG})$ is fabricated at the interface of silicon dioxide $\left(\mathrm{SiO}_{2}\right)$ and silicon via Chemical Vapor Deposition (CVD) method, herein for the first time. Carbon atoms bond with oxygen functionalities (i.e. $\mathrm{C}=\mathrm{O}, \mathrm{C}-\mathrm{OH}$ ) at the time of diffusion in $\mathrm{SiO}_{2}$ allowing for *Corresponding author. Tel: +86-0755-26525969. E-mail: fjstadler@szu.edu.cn (Florian J. Stadler)
\end{abstract}


$\mathrm{C} / \mathrm{O}$ ratios from $7 \sim 10$ adjustable by the variation of $\mathrm{SiO}_{2}$ thickness, indicating the tunable oxidation. Moreover, electronic structure and morphology of SOG are similar to the chemically grown rGO. The fabrication mechanism of SOG is also investigated.

\section{Introduction}

The carbon family consists of several allotropes from perfectly metal (i.e. graphene[1]) to insulator (i.e. diamond) as well as a handful of semiconducting carbon types such as carbon nanotubes (CNTs),[2, 3] graphene nanoribons,[4] graphene oxide (GO),[5] reduced graphene oxide (rGO)[6] and amorphous carbon (a-C).[7-9] Among these semiconducting carbon materials, GO is a two-dimensional (2D) semiconducting material, useful for several applications including photovoltaic devices,[10] batteries,[11] Raman enhancement,[12] fuel cells,[13] and supercapacitors.[14] rGO is obtained by chemically reducing GO to some extent by using suitable reducing agent e.g. hydrazine,[15] or vitamin-C[15]. GO and rGO both beneficial in several practical applications; however rGO is favorable in optoelectronic devices due to the elevated electrical and optical properties in comparison to GO.[16]

To date, the basic hurdle for the utilization of rGO in optoelectronic devices is the small sheet size that should be enhanced from micron size to centimeter scale production for many practical applications. Thus, several research groups are trying to produce large area rGO for example, Hasan et. al.[17] fabricated large area GO via electrophoretic deposition. On similar lines, Zhang et. al.[18] also synthesized rGO via this method for use as supercapacitor electrode. Guan et. al.[19] has developed a new method of the reduction of standing GO sheets via laser processing. Feng et al.[20] fabricated rGO via electronic solution reduction. Recently, Voiry et al.[21] reported high quality rGO via microwave reduction. However, the sheet size remained micron sized (i.e. $20 \sim 30 \mu \mathrm{m}$ ), indicating chemically produced rGO cannot be produced in large sheets due to the limitation of wet chemical process.[22, 23] 
Moreover, Ellmer[24] reported that carbon based semiconducting material as an active layer

\section{Experimental Methods}

\subsection{Chemical Synthesis of GO}

Graphite was oxidized by a modified Hummers' method.[25] In brief, $2 \mathrm{~g}$ of graphite flakes was added to a flask containing $50 \mathrm{~mL}$ of concentrated sulfuric acid. One gram of $\mathrm{NaNO}_{3}$ was added to the flask while stirring. Subsequently, $6 \mathrm{~g} \mathrm{KMnO}_{4}$ were poured slowly into the mixture in 20 min. to maintain the temperature under $20{ }^{\circ} \mathrm{C}$. The temperature was then increased to $35^{\circ} \mathrm{C}$ and kept at this condition for $1.5 \mathrm{~h}$. Then the mixture was cooled down to room temperature and left over night. Next, $400 \mathrm{~mL}$ of DI water was added to the mixture slowly. An amount of $50 \mathrm{~mL}$ of $\mathrm{H}_{2} \mathrm{O}_{2}$ solution was added drop wise to the solution. The reaction mixture was washed with $1.5 \mathrm{~L}$ of $\mathrm{HCl}(5 \mathrm{wt} \%$ in water), and then with deionized (DI) water (i.e. $1 \mathrm{~L} \times 3$ times) by using centrifugation at $4000 \mathrm{rpm}$. The paste obtained after centrifugation was added to $2 \mathrm{~L}$ of DI water and tip sonicated for $1 \mathrm{~h}$. After centrifugation 
and drying under vacuum, the graphene oxide (GO) was obtained as a brown colored powder.[26]

\subsection{Chemical Synthesis of rGO}

In a typical experiment, $0.1 \mathrm{~g}$ of graphene oxide (synthesized as described above) was dispersed in $50 \mathrm{~mL}$ DI water. Then $0.1 \mathrm{~mL}$ hydrazine monohydrate was added and maintained at $95{ }^{\circ} \mathrm{C}$ for $1 \mathrm{~h}$. Once the reaction is completed, the reduced graphene oxide (rGO) was collected by filtration as a black powder. The obtained material was washed with DI water several times to remove the excess hydrazine, and the final product was dried in a vacuum oven at $80{ }^{\circ} \mathrm{C}$.[27]

\subsection{Fabrication of SOG}

The rGO has synthesized by using CVD (GSL-1700X, MTI) method at the interface of a $\mathrm{SiO}_{2} / \mathrm{Si}$ substrate. A rectangular shaped (i.e. $1 \times 0.5 \mathrm{~cm}^{2}$ ) $\mathrm{SiO}_{2} / \mathrm{Si}$ was placed in an alumina boat and placed at the center of the CVD furnace tube. Three specimens $\mathrm{SiO}_{2}(\sim 100 \mathrm{~nm}) / \mathrm{Si}$, $\mathrm{SiO}_{2}(\sim 300 \mathrm{~nm})$ and $\mathrm{SiO}_{2}(\sim 500 \mathrm{~nm}) / \mathrm{Si}$ were used for the fabrication under similar growth conditions for comparison purposes.

Fabrication of SOG was done in five steps (Fig. 1 (a)). Firstly, the both ends of the furnace tube was sealed after the insertion of substrates and started a mechanical pump for creating a vacuum of $10^{-1}$ torr. Secondly, temperature was increased from room temperature to $100{ }^{\circ} \mathrm{C}$ at a rate of $5{ }^{\circ} \mathrm{C} / \mathrm{min}$ and a mixture of hydrogen/argon gas (i.e. $~ 25 \%$ of hydrogen mixed with $75 \%$ of argon) was provided throughout the experiment at the flow rate of $60 \mathrm{cc} / \mathrm{min}$ (centimeter cube per minute). Afterwards, the temperature was raised till $1100{ }^{\circ} \mathrm{C}$ at a rate of $10{ }^{\circ} \mathrm{C} / \mathrm{min}$. Thirdly, specimens were kept at $1100{ }^{\circ} \mathrm{C}$ for $10 \mathrm{~min}$. Fourthly, ethylene $\left(\mathrm{C}_{2} \mathrm{H}_{4}\right)$ was mixed with already provided mixture of gases at the rate of $100 \mathrm{cc} / \mathrm{min}$ for $5 \sim 15 \mathrm{~min}$ with maintaining $1100{ }^{\circ} \mathrm{C}$ and the $\mathrm{C}_{2} \mathrm{H}_{4}$ gas stopped mixing after particular growth time. Fifthly, the temperature of CVD furnace decreases in two steps, first step at a rate of 
$20{ }^{\circ} \mathrm{C} / \mathrm{min}$ till $500{ }^{\circ} \mathrm{C}$ and second step $5{ }^{\circ} \mathrm{C} / \mathrm{min}$ till room temperature. The specimens were

taken off from the furnace when temperature of CVD furnace reached at room temperature.

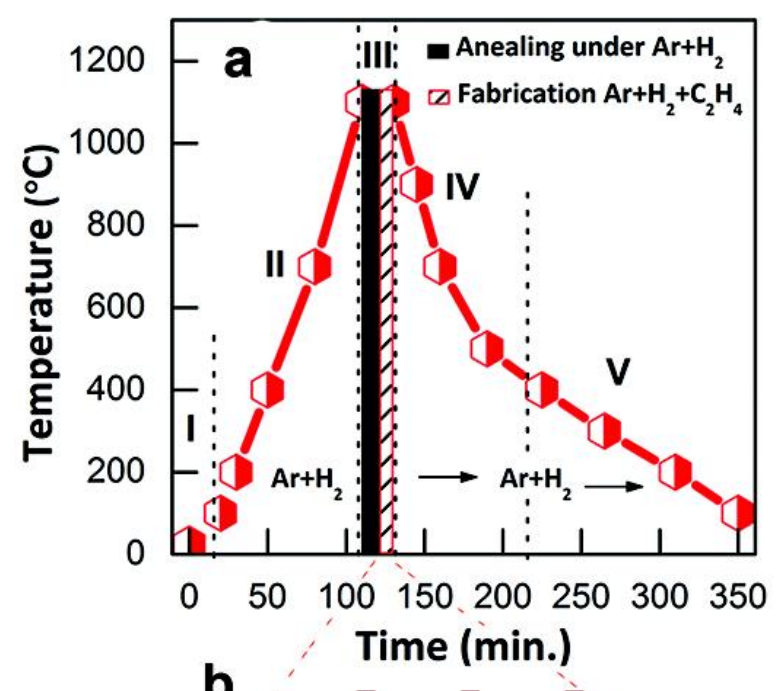

b

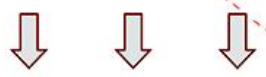

(c) (c) (c)

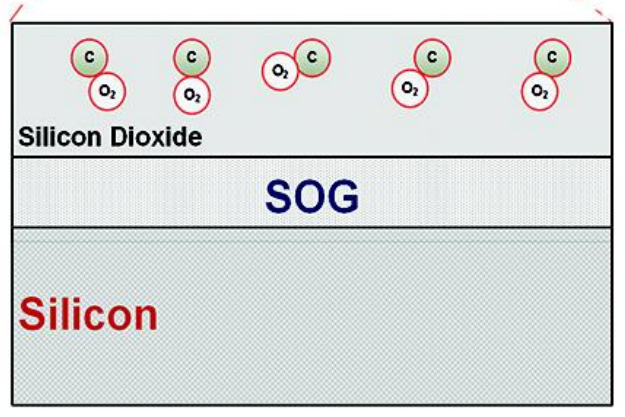

Fig. 1 (a) Growth conditions of SOG \& (b) Schematic of CVD-grown SOG at the interface of $\mathrm{SiO}_{2} / \mathrm{Si}$

The $\mathrm{C}-\mathrm{H}$ bond of $\mathrm{C}_{2} \mathrm{H}_{4}$ breaks at $1100{ }^{\circ} \mathrm{C}$ during the growth in CVD furnace and the resulting carbon atoms react with $\mathrm{SiO}_{2}$ to produce $\mathrm{SiC}$ and carbon monoxide. 


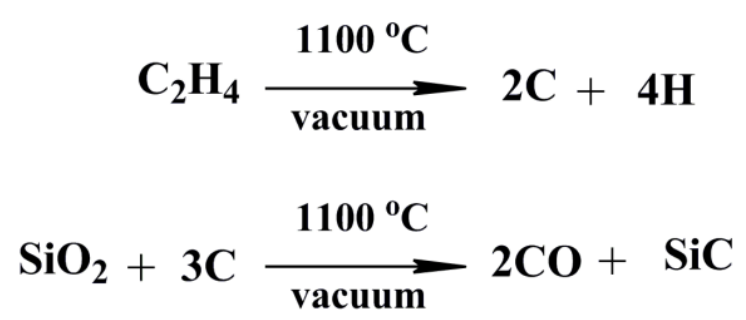

However, all the carbon atoms cannot be utilized forming this reaction. The oxygen vacancies are present in $\mathrm{SiO}_{2}$ that can make bonding with carbon atoms and travel towards the interface of $\mathrm{SiO}_{2} / \mathrm{Si}$. The thickness of $\mathrm{SiO}_{2}$ on the top of $\mathrm{Si}$ is varied from 100 to $500 \mathrm{~nm}$, act as a catalyst and oxygen donor (Fig. 1(b)). The carbon atoms were precipitated the surface of $\mathrm{SiO}_{2}$ and absorbed due to the amorphous structure of $\mathrm{SiO}_{2}$.[28] These carbon atoms diffuse through the $\mathrm{SiO}_{2}$ layer and reached at the interface of $\mathrm{SiO}_{2} / \mathrm{Si}$ and formed a thin layer of SOG.

\subsection{Transfer of SOG}

The specimens (i.e. $\mathrm{SiO}_{2} / \mathrm{SOG} / \mathrm{Si}$ ) were inserted into hydrofluoric (HF) acid for different amounts of time, depending the concentration of $\mathrm{HF}$ in the solution (Fig. 2). The $\mathrm{SiO}_{2}$ layer etched away, while SOG remains on the silicon. Then, acetone was poured into the solution to make it dilute. This step must do carefully, as layers of SOG can be lifted up in the solution easily before the removal of whole HF from the solution. The addition of large quantity of acetone makes the solution increasingly dilutes. However, HF cannot be removed completely with additional amount of acetone. To remove HF to a larger extent, some of the solution was removed and a similar amount of the acetone was added, simultaneously. In this way, the amount of HF in the solution decreases quickly. The removal of HF was observed by monitoring the $\mathrm{pH}$ of the solution. The specimens were kept in acetone at $\mathrm{pH} \sim 7$ overnight. 


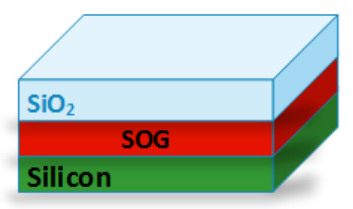

$\mathrm{SiO}_{2} / \mathrm{SOG} / \mathrm{Si}$

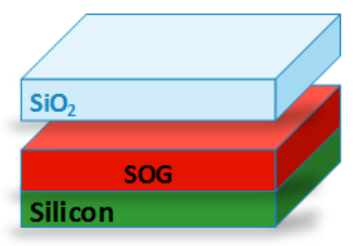

$\mathrm{HF}+\mathrm{SiO}_{2} / \mathrm{SOG} / \mathrm{Si}$

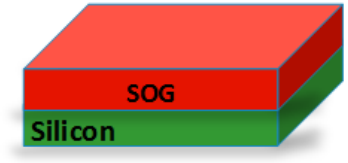

Removal of HF

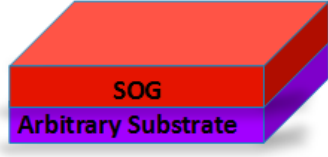

$\mathrm{SiO}_{2} / \mathrm{SOG} / \mathrm{Si}$

Fig. 2 Transfer of SOG on arbitrary substrate after removing $\mathrm{SiO}_{2} \sim 300 \mathrm{~nm}$ layer

\subsection{Characterization}

The SOG was characterized via high-resolution transmission electron microscope (HRTEM, JEOL-2010) and Raman spectroscopy (Renishaw-HR800) for structural analysis. Scanning electron microscopy (SEM-Hitachi, SU-70) and Atomic Force Microscopy (AFM, Shimadzu, SPM-9700) were used for surface \& morphology analyses. The chemical state of the surface atoms of SOG was investigated under X-ray photoelectron spectroscopy (XPS- ESCALAB $250 \mathrm{Xi}$ Thermo Scientific, USA). X-ray diffraction (XRD-Rigaku D/max 2500, $\lambda=1.5406$ $\AA$ ) was used for the phase of chemical grown rGO (chem-rGO) and SOG.

\section{Results and Discussion}

The successful fabrication of SOG via CVD was proven by several characterization tools after removing $\mathrm{SiO}_{2} \sim 300 \mathrm{~nm}$ layer. All specimens in this study were fabricated at the interface of $\mathrm{SiO}_{2} \sim 300 \mathrm{~nm}$ and silicon except specimens for the study of growth mechanism. The structure of SOG was investigated under HRTEM and wrinkles on the surface were observed (Fig. 3 (a)) as well as were observed in SEM images. These wrinkles are the feature of graphene and its derivatives. In the AFM images, we also observed similar feature (Fig. S1). Moreover, the $d$-spacing of SOG was similar to chemically produced rGO $\sim 0.36 \mathrm{~nm}$, indicating similar atomic and electronic structure to rGO (Fig. 3 (b)). Selected area 


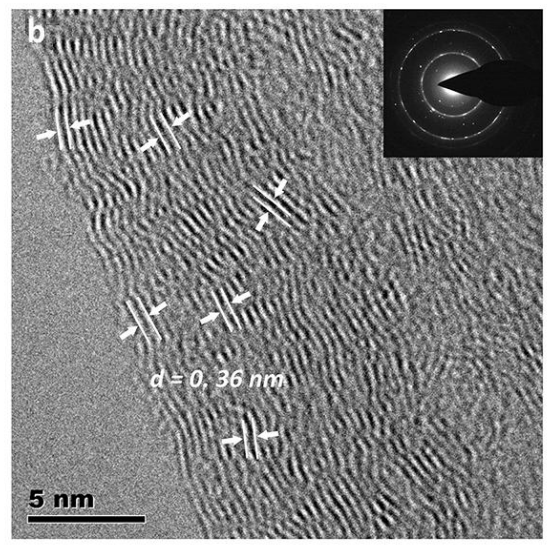

Fig. 3 (a) large area SOG \& (b) High-resolution image shows a d-spacing of $0.36 \mathrm{~nm}$

(inset: SAED).

Functional oxygen groups (e.g. hydroxy and epoxy etc.) are attached on the graphene surface during the chemical synthesis reaction, however non-oxygenated rings are also present in both GO and rGO (Fig. 5 (a)). XPS is a powerful tool for the observation of the amount of these oxygen functionalities as well as non-oxygenated rings. In XPS analysis of SOG, a peak at $287.9 \mathrm{eV}$ was observed, corresponding to the oxygenated rings and a peak at 284.6 eV corresponding to non-oxygenated rings (Fig. 5 (b)). Full width at half maximum of nonoxygenated rings was wider as compare to graphene, thus another peak of carbon and hydrogen $\mathrm{C}-\mathrm{H}$ can be fit well under it. This indicated that $\mathrm{C}-\mathrm{H}$ bonding was also present in 
SOG. These peaks in SOG are similar to the data found for chemically produced rGO. The $\mathrm{C} / \mathrm{O}$ ratio in SOG was around $\sim 8$, which is in close to the amount in chemically produced rGO.
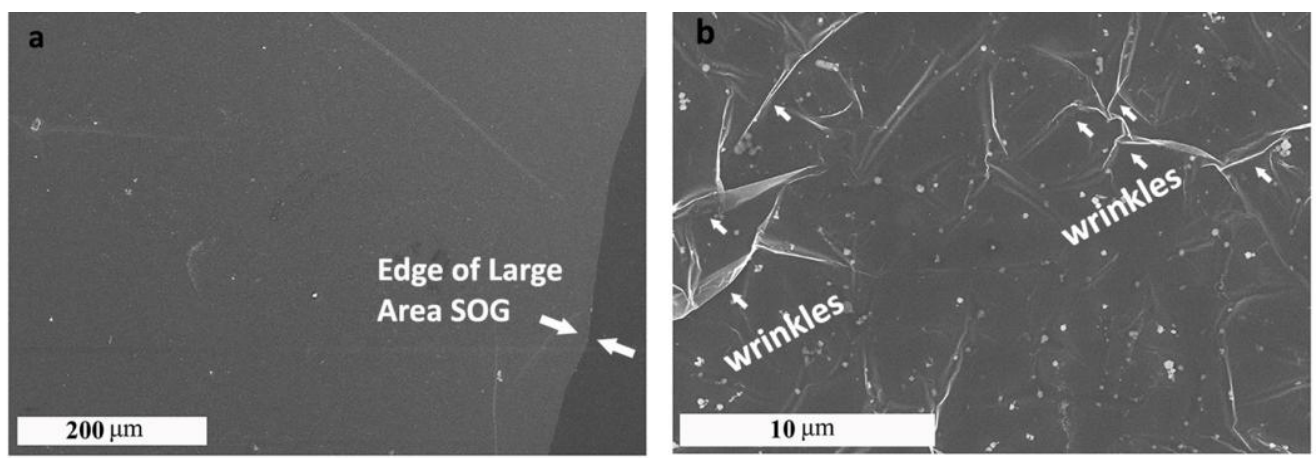

Fig. 4 (a) Large area transferred \& (b) Wrinkles on the surface of SOG that was fabricated by using a substrate of $\mathrm{SiO}_{2} \sim 300 \mathrm{~nm}$.

Two peaks were observed in the XRD signatures of chemically produced rGO at $21.6^{\circ}$ and $41.4^{\circ}$, indicating polycrystalline nature of chemically produced rGO (Fig. 5 (c)). On similar lines, XRD signature of SOG showed two peaks at different position $23.4^{\circ}$ and $40^{\circ}$, indicating slight oxidization. It is the fact that graphene peak was observed at $24^{\circ}$, which moved backward to $10^{\circ}$ in the case of $\mathrm{GO}$ and depending upon the oxidation it varied from 10 to $24.57^{\circ}$.[6] Moreover, two peaks at $23.4^{\circ}$ and $28^{\circ}$ can be fit under the wide XRD signature of SOG (Inset of Fig. 5 (c)). We believe that the peak fit at $28^{\circ}$ is due to the background effect of SOG/substrate, which did not appear in rGO XRD signature as it was in powder shape, while SOG was transferred on a substrate.

Four bands D, G, 2D and D+D' were observed in the Raman signature of chemically produced rGO as well as SOG (Fig. 5 (d)). The D band initiated due to the presence of defects and in perfectly crystalline graphene is almost absent. However, in the case of rGO and SOG, G band was intense, indicating these materials had higher structural defects in 
comparison to graphene. The $2 \mathrm{D}$ band is the overtone of these defects, which is very intense in graphene. It was intense in SOG case, while not intense for chemically produced rGO. The $\mathrm{G}$ band corresponded to the non-oxygenated rings, which was intense in SOG case due to thickness. The S3 band was also observed in both rGO and SOG.
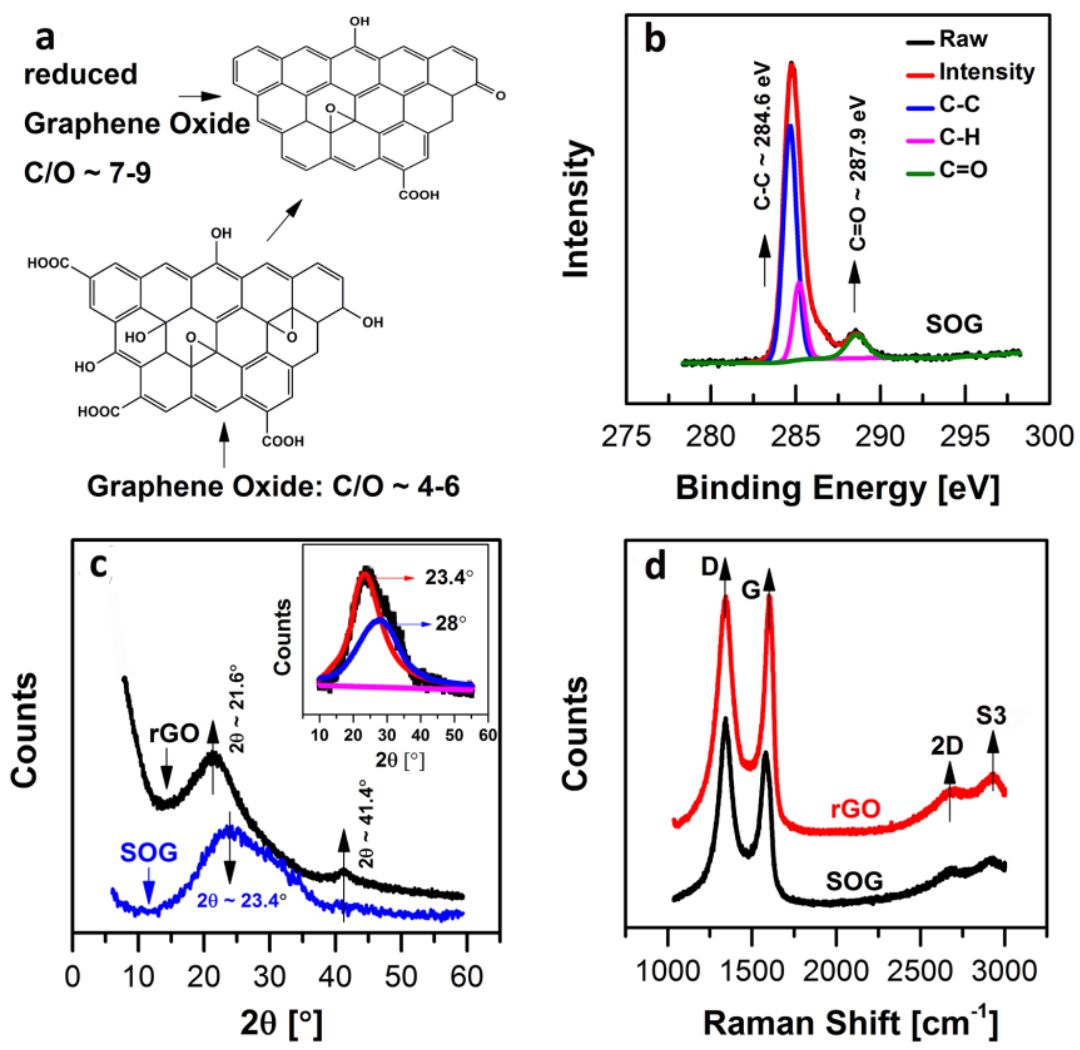

Fig. 5 (a) C/O ratio and graphene derivatives (i.e. GO \& rGO), (b) XPS spectra of SOG, (c) XRD signatures \& (d) Raman of chemically produced rGO and SOG (SOG specimen was fabricated by using $\mathrm{SiO}_{2} \sim 300 \mathrm{~nm}$ substrate)

The comparison of chemically produced rGO and SOG is summarized in Table. 1. Firstly, the fabrication method of SOG is CVD rather than Hummer's method or any other chemical method. Novoselov[30] described in his novel road map for the graphene that CVD is the best industrial fabrication method for the fabrication of cost affective and high quality graphene. Secondly, chemically produced rGO cannot have sheet size bigger than $20 \sim 30 \mu \mathrm{m}$, 
owing to the restriction of production method. Thirdly, C/O ratio of SOG can be tuned from 7 $\sim 10$, depending upon the thickness of $\mathrm{SiO}_{2}$ layer and it shows similar oxygen functional group in SOG as rGO other than C-O bond. Fourthly, XRD signatures of both SOG and chemical produced rGO show similar peak position. Fifthly, Raman spectrum shows four bands in both SOG and chemically produced rGO. Finally, the structure of chemically produced rGO and SOG is similar under HRTEM.

Table. 1 Comparison of chem-rGO \& SOG

\begin{tabular}{|c|c|c|}
\hline & Chem-rGO ${ }^{\text {Ref. }}$ & $\begin{array}{c}\text { SOG } \\
\text { (Present Study) }\end{array}$ \\
\hline Fabrication & Hummer's Method[25] & Chemical Vapor Deposition (CVD) \\
\hline \multirow[t]{2}{*}{ Method } & Improved Chemical Method[5] & \\
\hline & Other Methods[6, 19, 29, 31, 32] & \\
\hline Sheet size & $20 \sim 30 \mu \mathrm{m}[22,23]$ & $>200 \mu \mathrm{m}$ \\
\hline \multirow[t]{4}{*}{ XPS } & $\mathrm{C}-\mathrm{C} \sim 284.5 \mathrm{eV}[15,33]$ & $\mathrm{C}-\mathrm{C} \sim 284.6 \mathrm{eV}$ \\
\hline & $\mathrm{C}=\mathrm{O} \sim 287.8 \mathrm{eV}[15,33]$ & $\mathrm{C}=\mathrm{O} \sim 287.9 \mathrm{eV}$ \\
\hline & $\mathrm{C}-\mathrm{O} \sim 286.5 \mathrm{eV}[15,33]$ & --- \\
\hline & $\mathrm{C} / \mathrm{O}$ ratio $\sim 7-9[15,33]$ & $\mathrm{C} / \mathrm{O}$ ratio $\sim 7-10$ \\
\hline XRD & $2 \theta \sim 10.27$ to $24.57^{\circ}[6]$ & $2 \theta \sim 23.4^{\circ}(\mathrm{SOG})$ \\
\hline \multirow[t]{4}{*}{ Raman } & $\mathrm{D}$ band[6] $\sim 1334 \mathrm{~cm}^{-1}$ & $\mathrm{D}$ band $\sim 1341 \mathrm{~cm}^{-1}$ \\
\hline & $\mathrm{G}$ band $[6] \sim 1581 \mathrm{~cm}^{-1}$ & $\mathrm{G}$ band $\sim 1581 \mathrm{~cm}^{-1}$ \\
\hline & $2 \mathrm{D}$ band $[6] \sim 2700 \mathrm{~cm}^{-1}$ & 2D band $\sim 2700 \mathrm{~cm}^{-1}$ \\
\hline & $\mathrm{S} 3$ band[6] $2910 \mathrm{~cm}^{-1}$ & $\mathrm{~S} 3$ band $\sim 2910 \mathrm{~cm}^{-1}$ \\
\hline HRTEM & $\mathrm{d}$-spacing $[6] \sim 0.36 \mathrm{~nm}$ & d-spacing $\sim 0.36 \mathrm{~nm}$ \\
\hline
\end{tabular}


The growth mechanism of SOG was also investigated with the help of XPS. The C/O ratio can be tuned with the help of thickness of $\mathrm{SiO}_{2}$. As $\mathrm{SiO}_{2} \sim 300 \mathrm{~nm} / \mathrm{Si}$ is amorphous in nature and at high temperature dangling bonds start $\mathrm{OH}$ bonding at the surface of $\mathrm{SiO} 2$ due to the ability of hydrogen gas. Hao et. al.[34] reported that $\mathrm{OH}$ bond is more favorable for the fabrication of large area graphene with the help of density functional theory (DFT). They described that hydrogen terminated edges of the graphene limits the expansion of the monolayer graphene sheet. In the viewpoint of this study, $\mathrm{OH}$ bond is useful for the fabrication of large area graphene and presence of this bond in SOG may help for the fabrication of large area. Moreover, Chen et. al.[28] successfully fabricated graphene on the top of $\mathrm{SiO}_{2}$ substrate with the help of oxygen as well as described that carbon atoms can diffuse into $\mathrm{SiO}_{2}$. However, they obtained graphene rather than oxidized graphene and, thus, their results cannot be compared with SOG. However, we can expect that oxygen plays an important role for the production of large area graphene and carbon atoms can diffuse into $\mathrm{SiO}_{2}$. 


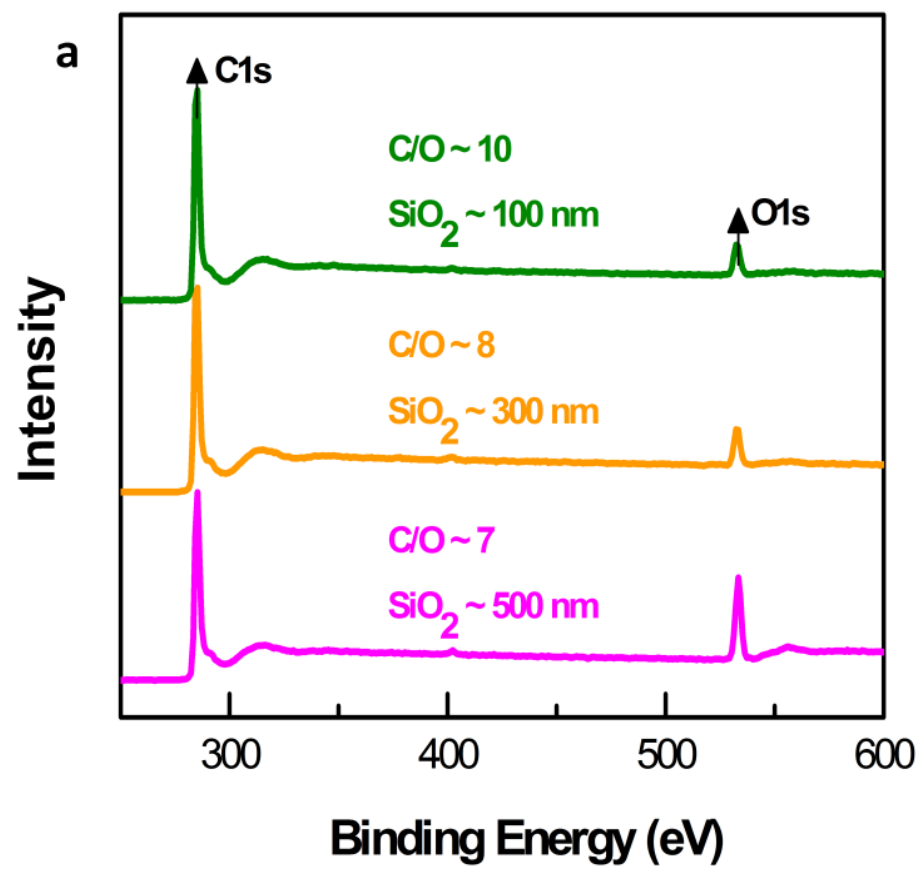

b

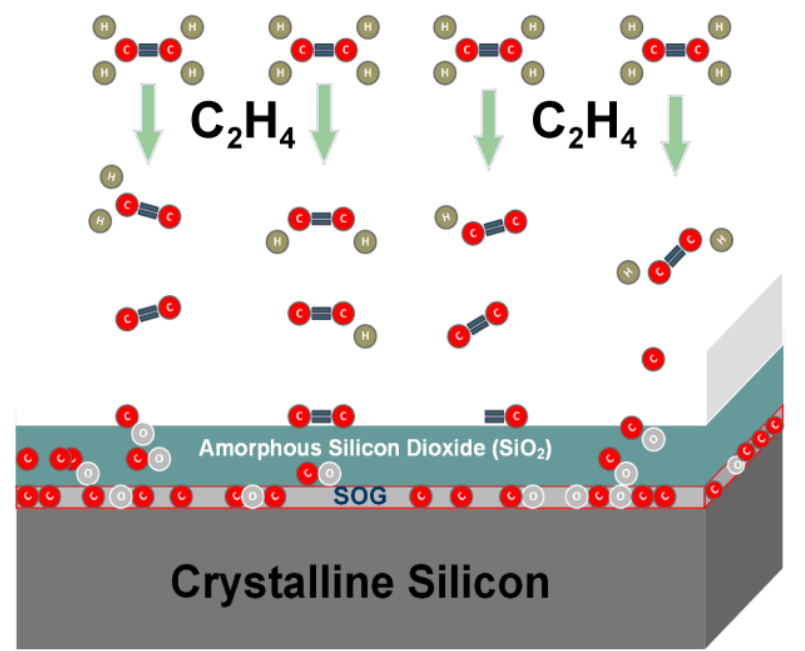

Fig. 6 (a) XPS survey of SOG fabricated by using different thick $\mathrm{SiO}_{2}$ layer $(100 \sim 500$ nm). (b) Schematic of SOG fabrication

Therefore, CVD is used for the fabrication of SOG without adding oxygen while using $\mathrm{SiO}_{2}$ as a catalyst and oxygen donor. Therefore, three specimens of $\mathrm{SiO}_{2}, 100,300$ and $500 \mathrm{~nm}$ are used as catalyst and oxygen donor. The XPS analysis of the product from these three specimens shows significant difference in $\mathrm{C} / \mathrm{O}$ ratio, indicating tunable oxygen impurities 
depending upon the thickness of $\mathrm{SiO}_{2}$ (Fig. 6 (a)). The carbon atoms travel from top to bottom of $\mathrm{SiO}_{2}$ and reach at the interface of $\mathrm{SiO}_{2} /$ silicon (Fig. 6 (b)). The carbon atoms cannot diffuse more due to the perfectly crystalline silicon surface. However, these carbon atoms bring oxygen impurities from $\mathrm{SiO}_{2}$ during their flow from top to bottom. Thus, these carbon atoms are collected at the interface and reduction of temperature forces them to lose the energy. These molecules start forming bonds with each other and subsequently an ultrathin layer of SOG is formed.

\section{Conclusions}

Herein, we reported successful fabrication of centimeter scale SOG via CVD for the first time according to the best of our knowledge. Several chemical based fabrication methods for the preparation of rGO are favorable among the research groups to produce large area oxidized graphene. However, these methods could not produce larger than micron size as these methods are using wet chemical strategy, which is not useful for producing centimeter scale oxidized graphene. Therefore, we used $\mathrm{SiO}_{2}$ layers $(100 \sim 500 \mathrm{~nm})$ as a catalyst and oxygen donor for the production of SOG by using an industrial production method CVD. Moreover, we produced chemically GO and reduced it via hydrazine for comparison purposes. The SOG is analyzed with different characterization tools and found similar morphology. HRTEM results clearly demonstrate that SOG is similar to chemically produced rGO. Raman and XRD signatures also were compared with chemically produced rGO and similarities were found. The electronic structure of SOG is similar to rGO and we believe that it can be very useful material in optoelectronic materials. In addition, the production of centimeter size SOG will be beneficial as an active material in transparent conducting electrode (TCE). 


\section{Acknowledgement}

The authors would like to thank the National Science Foundation of China (21574086), Nanshan District Key Lab for Biopolymers and Safety Evaluation (No. KC2014ZDZJ0001A), Shenzhen Sci \& Tech research grant (ZDSYS201507141105130), Shenzhen City Science and Technology Plan Project (JCYJ20140509172719311). R. U. R. Sagar would like to thank the Postdoctoral Science Foundation of China (No. 2016M592531) for financial support.

\section{Author's Contribution}

R. U. R. Sagar has fabricated, observed, and explained the SOG. F. J. Stadler helped in composing and analyzing the data. Moreover, he is head of the group and as such responsible for research funding and lab facilities for all experiments. The remaining co-authors helped in data interpretation. 


\section{References}

[1] R.U.R. Sagar, N. Mahmood, F.J. Stadler, T. Anwar, S.T. Navale, K. Shehzad, B. Du, High Capacity Retention Anode Material for Lithium Ion Battery, Electrochim Acta 211 (2016) 156-163.

[2] R. Zhang, Y. Zhang, Q. Zhang, H. Xie, W. Qian, F. Wei, Growth of Half-Meter Long Carbon Nanotubes Based on Schulz-Flory Distribution, Acs Nano 7(7) (2013) 6156-6161.

[3] K. Shehzad, Z.-M. Dang, M.N. Ahmad, R.U.R. Sagar, S. Butt, M.U. Farooq, T.-B. Wang, Effects of carbon nanotubes aspect ratio on the qualitative and quantitative aspects of frequency response of electrical conductivity and dielectric permittivity in the carbon nanotube/polymer composites, Carbon 54 (2013) 105-112.

[4] J. Bai, R. Cheng, F. Xiu, L. Liao, M. Wang, A. Shailos, K.L. Wang, Y. Huang, X. Duan, Very large magnetoresistance in graphene nanoribbons, Nat Nano 5(9) (2010) 655-659.

[5] D.C. Marcano, D.V. Kosynkin, J.M. Berlin, A. Sinitskii, Z. Sun, A. Slesarev, L.B. Alemany, W. Lu, J.M. Tour, Improved Synthesis of Graphene Oxide, ACS Nano 4(8) (2010) 4806-4814.

[6] I.K. Moon, J. Lee, R.S. Ruoff, H. Lee, Reduced graphene oxide by chemical graphitization, Nature Communications 1 (2010) 73.

[7] R.U.R. Sagar, A.S. Saleemi, X. Zhang, Angular magnetoresistance in semiconducting undoped amorphous carbon thin films, Journal of Applied Physics 117(17) (2015) 174503.

[8] R.U.R. Sagar, X.Z. Zhang, C.Y. Xiong, Y. Yu, Semiconducting amorphous carbon thin films for transparent conducting electrodes, Carbon 76 (2014) 64-70.

[9] R. Ur Rehman Sagar, X. Zhang, J. Wang, C. Xiong, Negative magnetoresistance in undoped semiconducting amorphous carbon films, J Appl Phys 115(12) (2014) 123708.

[10] D. Konios, G. Kakavelakis, C. Petridis, K. Savva, E. Stratakis, E. Kymakis, Highly efficient organic photovoltaic devices utilizing work-function tuned graphene oxide derivatives as the anode and cathode charge extraction layers, J Mater Chem A 4(5) (2016) $1612-1623$.

[11] C. Zhao, H. Gao, C. Chen, H. Wu, Reduction of graphene oxide in Li-ion batteries, J Mater Chem A 3(36) (2015) 18360-18364.

[12] Q. Liu, L. Wei, J. Wang, F. Peng, D. Luo, R. Cui, Y. Niu, X. Qin, Y. Liu, H. Sun, J. Yang, Y. Li, Cell imaging by graphene oxide based on surface enhanced Raman scattering, Nanoscale 4(22) (2012) 7084-7089.

[13] E.S. Cho, A.M. Ruminski, S. Aloni, Y.-S. Liu, J. Guo, J.J. Urban, Graphene oxide/metal nanocrystal multilaminates as the atomic limit for safe and selective hydrogen storage, Nat Commun 7 (2016) 10804.

[14] X. Cui, R. Lv, R.U.R. Sagar, C. Liu, Z. Zhang, Reduced graphene oxide/carbon nanotube hybrid film as high performance negative electrode for supercapacitor, Electrochimica Acta 169 (2015) 342-350.

[15] S. Pei, H.-M. Cheng, The reduction of graphene oxide, Carbon 50(9) (2012) 3210-3228. [16] F. Bonaccorso, Z. Sun, T. Hasan, A.C. Ferrari, Graphene photonics and optoelectronics, Nat Photon 4(9) (2010) 611-622.

[17] S.A. Hasan, J.L. Rigueur, R.R. Harl, A.J. Krejci, I. Gonzalo-Juan, B.R. Rogers, J.H. Dickerson, Transferable Graphene Oxide Films with Tunable Microstructures, Acs Nano 4(12) (2010) 7367-7372.

[18] H. Zhang, X. Zhang, D. Zhang, X. Sun, H. Lin, C. Wang, Y. Ma, One-Step Electrophoretic Deposition of Reduced Graphene Oxide and $\mathrm{Ni}(\mathrm{OH}) 2$ Composite Films for Controlled Syntheses Supercapacitor Electrodes, The Journal of Physical Chemistry B 117(6) (2013) 1616-1627. 

reduced Graphene Oxide in Liquid Nitrogen Environment, Sci Rep 6 (2016) 28913.

[20] H. Feng, R. Cheng, X. Zhao, X. Duan, J. Li, A low-temperature method to produce highly reduced graphene oxide, Nat Commun 4 (2013) 1539.

[21] D. Voiry, J. Yang, J. Kupferberg, R. Fullon, C. Lee, H.Y. Jeong, H.S. Shin, M. Chhowalla, High-quality graphene via microwave reduction of solution-exfoliated graphene oxide, Science (2016).

[22] S. Pan, I.A. Aksay, Factors Controlling the Size of Graphene Oxide Sheets Produced via the Graphite Oxide Route, Acs Nano 5(5) (2011) 4073-4083.

[23] K.E. Lee, J.E. Kim, U.N. Maiti, J. Lim, J.O. Hwang, J. Shim, J.J. Oh, T. Yun, S.O. Kim, Liquid Crystal Size Selection of Large-Size Graphene Oxide for Size-Dependent N-Doping and Oxygen Reduction Catalysis, Acs Nano 8(9) (2014) 9073-9080.

[24] K. Ellmer, Past achievements and future challenges in the development of optically transparent electrodes, Nat Photon 6(12) (2012) 809-817.

[25] W.S. Hummers, R.E. Offeman, Preparation of Graphitic Oxide, J Am Chem Soc 80(6) (1958) 1339-1339.

[26] N.D. Luong, L.H. Sinh, L.-S. Johansson, J. Campell, J. Seppälä, Functional Graphene by Thiol-ene Click Chemistry, Chemistry - A European Journal 21(8) (2015) 3183-3186.

[27] K. Zhang, L.L. Zhang, X.S. Zhao, J. Wu, Graphene/Polyaniline Nanofiber Composites as Supercapacitor Electrodes, Chem Mater 22(4) (2010) 1392-1401.

[28] J. Chen, Y. Wen, Y. Guo, B. Wu, L. Huang, Y. Xue, D. Geng, D. Wang, G. Yu, Y. Liu, Oxygen-Aided Synthesis of Polycrystalline Graphene on Silicon Dioxide Substrates, J Am Chem Soc 133(44) (2011) 17548-17551.

[29] M.M. Jaafar, G.P.M.K. Ciniciato, S.A. Ibrahim, S.M. Phang, K. Yunus, A.C. Fisher, M. Iwamoto, P. Vengadesh, Preparation of a Three-Dimensional Reduced Graphene Oxide Film by Using the Langmuir-Blodgett Method, Langmuir 31(38) (2015) 10426-10434.

[30] K.S. Novoselov, V.I. Fal'ko, L. Colombo, P.R. Gellert, M.G. Schwab, K. Kim, A roadmap for graphene, Nature 490(7419) (2012) 192-200.

[31] S. Abdolhosseinzadeh, H. Asgharzadeh, H. Seop Kim, Fast and fully-scalable synthesis of reduced graphene oxide, Sci Rep 5 (2015) 10160.

[32] R. Flyunt, W. Knolle, A. Kahnt, C.E. Halbig, A. Lotnyk, T. Haupl, A. Prager, S. Eigler, B. Abel, High quality reduced graphene oxide flakes by fast kinetically controlled and clean indirect UV-induced radical reduction, Nanoscale 8(14) (2016) 7572-9.

[33] D.R. Dreyer, S. Park, C.W. Bielawski, R.S. Ruoff, The chemistry of graphene oxide, Chem Soc Rev 39(1) (2010) 228-40.

[34] Y. Hao, M.S. Bharathi, L. Wang, Y. Liu, H. Chen, S. Nie, X. Wang, H. Chou, C. Tan, B. Fallahazad, H. Ramanarayan, C.W. Magnuson, E. Tutuc, B.I. Yakobson, K.F. McCarty, Y.W. Zhang, P. Kim, J. Hone, L. Colombo, R.S. Ruoff, The Role of Surface Oxygen in the Growth of Large Single-Crystal Graphene on Copper, Science 342(6159) (2013) 720. 


\section{Figure Captions}

Fig. 1 (a) Growth conditions of SOG \& (b) Schematic of CVD-grown SOG at the interface of $\mathrm{SiO}_{2} / \mathrm{Si}$

Fig. 2 Transfer of SOG on arbitrary substrate after removing $\mathrm{SiO}_{2} \sim 300 \mathrm{~nm}$ layer

Fig. 3 (a) large area SOG \& (b) High-resolution image shows a d-spacing of $0.36 \mathrm{~nm}$ (inset: SAED).

Fig. 4 (a) Large area transferred \& (b) Wrinkles on the surface of SOG that was fabricated by using a substrate of $\mathrm{SiO}_{2} \sim 300 \mathrm{~nm}$.

Fig. 5 (a) C/O ratio and graphene derivatives (i.e. GO \& rGO), (b) XPS spectra of SOG, (c) XRD signatures \& (d) Raman of chemically produced rGO and SOG (SOG specimen was fabricated by using $\mathrm{SiO}_{2} \sim 300 \mathrm{~nm}$ substrate)

Fig. 6 (a) XPS survey of SOG fabricated by using different thick $\mathrm{SiO}_{2}$ layer $(100 \sim 500 \mathrm{~nm})$.

(b) Schematic of SOG fabrication 

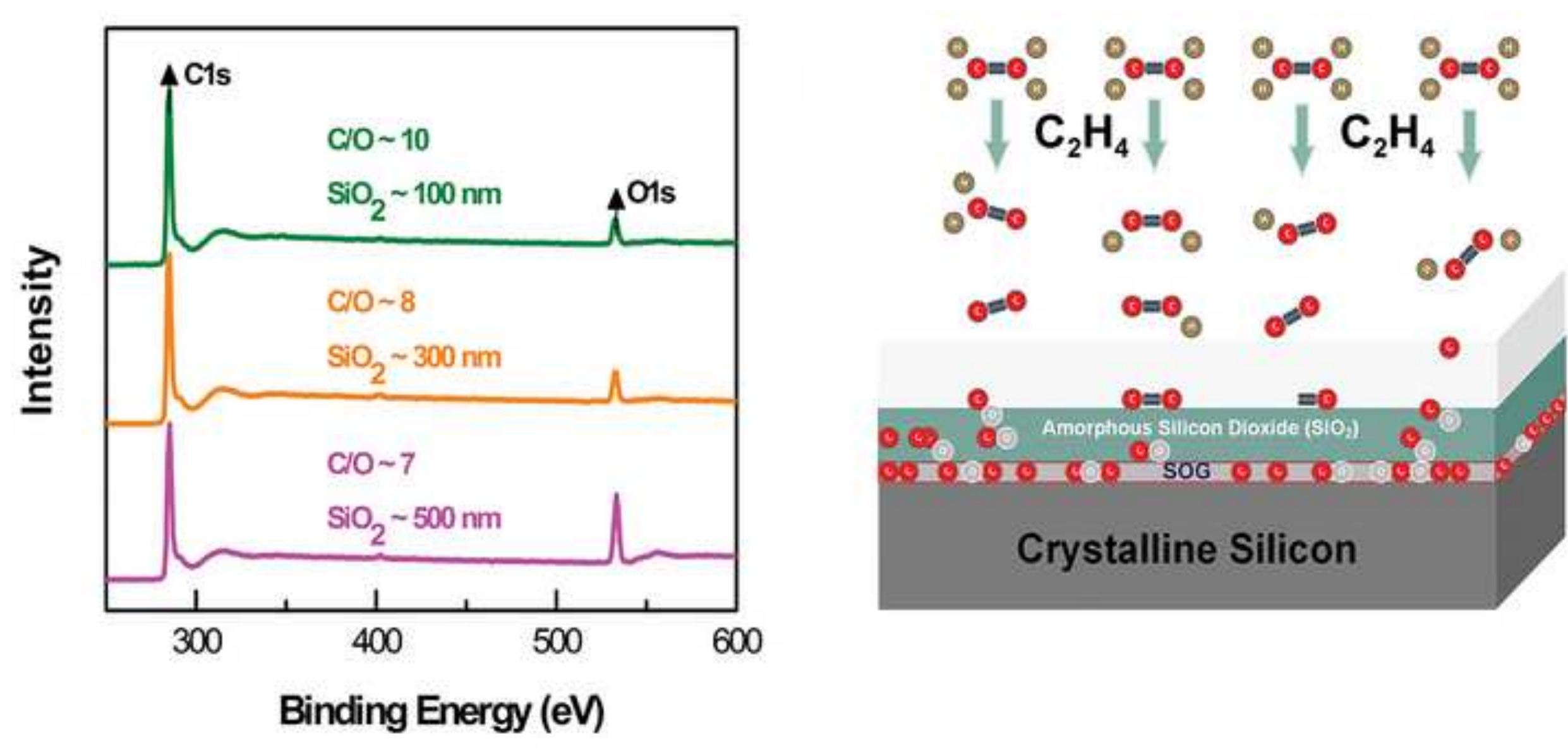

Binding Energy (eV)

\section{Crystalline Silicon}

